\title{
Enseñanza de vocabulario en inglés a estudiantes de educación superior técnico profesional. Lengua de señas chilena como estrategia multimodal
}

Teaching English Vocabulary to Higher Technical Professional Education Students. Chilean Sign Language as a Multimodal Strategy

Ensino de vocabulário em inglês a alunos do ensino superior técnico e profissional. Linguagem de sinais chilena como estratégia multimodal

María Francisca Lohaus Reyes https://orcid.org/0000-0002-I207-087X Universidad de Chile, Chile mlohaus@ug.uchile.cl

Valeria Herrera-Fernández https://orcid.org/oooo-oooi-8I76-6195 Universidad de Chile, Chile valherrera@u.uchile.cl

Received: 26/03/2020

Accepted by peers: 04/11/2020
Sent to peer review: 14/04/2020

Approved: 21/04/2021

DOI: 10.5294/laclil.2021.14.1.4

To reference this article (APA) / Para citar este artículo (APA) / Para citar este artigo (APA) Lohaus, M. F., \& Herrera-Fernández, V. (2021). Enseñanza de vocabulario en inglés a estudiantes de educación superior técnico profesional. Lengua de eñas chilena como estrategia multimodal. Latin American Journal of Content \& Language Integrated Learning, 14(1), 99-122. https://doi.org/10.5294/laclil.2021.14.1.4 
RESUMEN. Los adultos chilenos poseen bajo dominio del idioma inglés respecto a sus pares de la Organización para la Cooperación y el Desarrollo Económico (OCDE). Para mejorar estos resultados, es necesario diversificar las metodologías de enseñanza de dicha lengua, especialmente en la Educación Superior Técnico Profesional (ESTP). El objetivo del estudio fue determinar el efecto de una estrategia didáctica multimodal para la enseñanza de vocabulario oral aislado en inglés, usando lengua de señas chilena (LSCh) y alfabeto manual. Se utilizó una metodología cuantitativa y un diseño cuasi experimental con pre y postest. Se conformaron dos grupos de 30 participantes cada uno, con edades entre 16 y 44 años, a quienes se evaluó el vocabulario productivo oral. El grupo experimental recibió la estrategia didáctica diseñada y el grupo control recibió enseñanza tradicional. Los análisis realizados concluyen que la estrategia implementada en el grupo experimental produjo un efecto positivo en la producción oral de vocabulario, comparado con el grupo control.

Palabras clave (Fuente: tesauro de la Unesco): didáctica; enseñanza de lenguas; enseñanza del inglés como lengua extranjera; lengua de señas; educación de adultos.

ABSTRACT. Chilean adults report a low level of English proficiency compared to their OECD peersFor this reason, the teaching methodologies of this foreign language should become more relevant in tertiary education, especially in Higher Technical Professional Education (ESTP). The research objective was to determine the effect of a didactic strategy for teaching English vocabulary that incorporates the use of Chilean Sign Language and the manual alphabet in the production of isolated oral vocabulary in ESTP students. A quantitative methodology and a quasi-experimental design were used in the study. Two groups of 30 people between the ages of 16 and 44 participated, to whom an instrument was applied to evaluate the oral productive vocabulary in four categories of words. One of the groups underwent the designed multimodal teaching strategy and the other group received traditional vocabulary instruction by oral repetition. After the intervention, both groups were evaluated with the same instrument. Nonparametric analyses concluded that the multimodal teaching strategy implemented with the experimental group produced a shortterm positive effect on the oral production of the isolated vocabulary of the students, as compared to the control group.

Keywords (Source: Unesco Thesaurus): teaching approach; language teaching; teaching English as a foreign language; sign language; adult education.

RESUMO. Adultos chilenos têm baixo domínio da língua inglesa em comparação com seus pares da Organização para Cooperação e Desenvolvimento Econômico (OCDE). Para melhorar estes resultados, é necessário diversificar as metodologias de ensino desta língua, especialmente na Educação Profissional Técnico Superior (ESTP). O objetivo do estudo foi determinar o efeito de uma estratégia didática multimodal para o ensino de vocabulário oral isolado em inglês, utilizando a língua de sinais chilena (LSCh) e o alfabeto manual. Foi usada uma metodologia quantitativa e um desenho quase experimental com pré e pós-teste. Foram formados dois grupos de 30 participantes cada um, com idades entre 16 e 44 anos, que foram avaliados quanto ao vocabulário produtivo oral. 0 grupo experimental recebeu a estratégia didática elaborada e o grupo controle recebeu o ensino tradicional. As análises realizadas concluem que a estratégia implementada no grupo experimental produziu efeito positivo na produção oral do vocabulário, em comparação ao grupo controle.

Palavras-chave (Fonte: tesauro da Unesco): didática; ensino de línguas; ensino de inglês como língua estrangeira; linguagem de sinais; educação de adultos. 


\section{Introducción}

La enseñanza del inglés en el sistema educativo formal ha adquirido especial relevancia en la última década, con énfasis en metodologías que incentiven el aprendizaje de esta lengua. Según el Ministerio de Educación de Chile (2019; 2016), la Educación Superior Técnico Profesional (ESTP), estimulada por las necesidades del contexto global, busca desarrollar competencias comunicativas en inglés en los programas de formación de los futuros profesionales técnicos del país.

El estudio se origina en la necesidad de proveer metodologías de enseñanza multimodales para el idioma inglés como lengua extranjera, sensibles a contextos específicos y diversos de la ESTP y que respondan a las demandas de inclusión que la sociedad realiza al sistema educativo (Ministerio de Educación de Chile, 2009). El diseño es cuasi experimental y su objetivo es determinar el efecto de una estrategia de enseñanza multimodal basada en la incorporación de lengua de señas chilena (LSCh) y deletreo manual (dactilología) en la producción oral de vocabulario básico aislado en inglés en estudiantes de ESTP.

El estudio se justifica debido a que las influencias de la globalización y las necesidades del mercado laboral son claramente visibles en la educación chilena. Al respecto, Zajda (2018) plantea que el cambio tecnológico y la apertura hacia la economía global llevan a un replanteamiento de los conocimientos que las sociedades deben enseñar y aprender, y esto se ha proyectado a todos los niveles educativos. La ESTP diseña su oferta curricular según los lineamientos que entrega el sector productivo y de servicios, para dar respuesta a sus necesidades de personal técnico capacitado (Ministerio de Educación de Chile, 2016). Esta formación se da a través de un currículo por competencias, definidas como un conjunto de habilidades y conocimientos necesarios para llevar a cabo una tarea (Zabalza, 2003). Dentro de estas competencias, se considera el dominio del inglés como idioma extranjero. En Chile, el 77\% de los Centros de Formación Técnica (CFT) y los Institutos Profesionales (IP) acreditados incluyen el idioma inglés en sus mallas curriculares (Vertebral, 2018).

El reporte 2019, realizado por la organización English First, advierte la necesidad de que los adultos desarrollen habilidades comunicativas 
sólidas en inglés para transitar hacia una economía basada en el conocimiento, el desarrollo tecnológico y la colaboración en el escenario científico internacional. Este reporte clasifica a Chile con un nivel moderado y lo sitúa en el lugar 42 del ránking mundial y en séptimo lugar en América Latina. Junto con ello, el informe del British Council (2015) distingue la existencia de problemas estructurales del sistema educativo en Chile, como la baja comprensión lectora en la lengua materna, que impactan la enseñanza y el aprendizaje del inglés.

Los resultados de ambos informes se relacionan con los obtenidos en el Sistema de Medición de la Calidad de la Educación (SIMCE) de inglés, donde menos del $20 \%$ de los estudiantes de tercer año de educación secundaria del país presentan dominio básico (A2) o pre intermedio comunicativo (B1) del idioma, según el Marco Común Europeo de Referencia de Idiomas. La consistencia de los bajos resultados en las evaluaciones estandarizadas nacionales e internacionales (British Council, 2015; Ministerio de Educación de Chile, 2013a; 2013b) muestra que lograr fluidez comunicativa y comprensiva en inglés en las aulas chilenas es difícil y conlleva afrontar múltiples desafíos a largo plazo. Considerando lo expuesto, la enseñanza del inglés para adultos en la educación chilena se sitúa en un escenario exigente para los actores involucrados.

En este sentido, para afrontar los desafíos en torno a la enseñanza del inglés como lengua extranjera en Chile, desde los años ochenta se comienzan a investigar las metodologías utilizadas en el país, transitando entre la presentación explícita de los mecanismos gramaticales y tareas repetitivas-memorísticas de corte conductista, hacia un enfoque comunicativo que favorece la expresión de ideas, más que el énfasis en los aspectos sintáctico-gramaticales (McBride, 2009; Richards \& Rodgers, 2001). Como señalan Kumaravadivelu (2003) y LarsenFreeman (2004), se requiere lograr un equilibrio entre ambos enfoques y seleccionar las metodologías que mejor funcionen en el contexto específico, de manera que los estudiantes utilicen la lengua para sus propios propósitos, considerando sus características, necesidades y expectativas personales.

Desde esta perspectiva, resulta fundamental afirmar que las personas tienen distintas percepciones y sensaciones al enfrentarse al aprendizaje de una lengua extranjera (McBride, 2009). Por este motivo, 
es necesario asegurar que todos los estudiantes comprendan los mensajes con un acercamiento integral, atractivo y comprensible, con el fin de lograr aprendizajes significativos (Krashen, 2002). Para conseguir ese objetivo, el profesor debe aproximar la experiencia de la lengua extranjera a sus estudiantes, a partir de su conocimiento pedagógico y el contacto permanente con los educandos (Richards \& Rodgers, 2001), vinculando sus necesidades y estilos de aprendizaje con prácticas y estrategias situadas de enseñanza.

En este acercamiento, se deben eliminar barreras físicas, sensoriales y cognitivas para ofrecer oportunidades de aprendizaje reales a todos, sin importar su forma de acceso al conocimiento (Echeita \& Ainscow, 2011). Para lograr ese objetivo, la multimodalidad, entendida como la utilización de diferentes modalidades conectadas con los cinco sentidos (Castro \& Rodríguez, 2017), ofrece una alternativa interesante para diseñar estrategias metodológicas de enseñanza de la lengua extranjera, especialmente en la ESTP, porque se reconoce la importancia del movimiento, el contacto visual y los estímulos sonoros y visuales en el aprendizaje (Grinder \& Bandler, 1998; Thornbury, 2002).

El diseño instruccional utilizado mayoritariamente en las aulas de lengua extranjera de educación superior suele ser insuficiente para lograr competencia comunicativa oral. Al respecto, McKay (2003) señala que la mayoría de los profesores no utiliza estrategias de trabajo grupal u oral-comunicativas, debido al gran número de estudiantes en sala, que complejiza la evaluación de esas habilidades, y al rechazo de los estudiantes a hablar en inglés en público. Según el British Council (2015), este rechazo se puede atribuir a la timidez, que dificulta la oralización en la lengua extranjera por vergüenza o miedo. Lo anterior se suma a otros factores, como la motivación de estudiantes y profesores, contextos monótonos en el aula y falta de materiales multimodales (Syukri \& Humaera, 2019; Vera, 2008). Debido a que la voluntad de comunicación en una segunda lengua está influida por los diversos factores ya mencionados, el estudio pretendió afrontar aquellos que afectan la oralización en la segunda lengua. Para ello, se propuso un diseño instruccional que aumentara los medios de expresión, con el fin de promover la oralidad en idioma inglés.

Las actividades planificadas para la enseñanza del inglés en el contexto chileno utilizan, en su mayoría, medios de representación 
principalmente visuales y auditivos (Brown, 2000; McBride, 2009; Vera, 2008). Sin embargo, al ignorar otros modos de representación, se restringe el entorno de aprendizaje para la expresión oral en la lengua extranjera al limitar el acceso físico y sensorial al léxico mental (Hotstetter et al., 2007; Krauss et al., 2000), lo que dificulta el rescate de las palabras en la segunda lengua y su posterior expresión oral.

Méndez y Peña (2013) señalan que los/las estudiantes de una lengua extranjera requieren compromiso e inmersión física, intelectual y emocional para aprenderla. Por su parte, García y Cole (2014) argumentan que las lenguas no son sistemas autónomos que las personas poseen, sino prácticas que se ejercen día a día en un mundo plurilingüe en el que la meta es la comunicación. Las autoras reconocen que los códigos lingüísticos son susceptibles de mezclarse para la concreción de propósitos específicos.

Al respecto, la Teoría de Interdependencia Lingüística (TIL), formulada por Cummins (2002; 2006), postula que el conocimiento metacognitivo, conceptual, lingüístico, metalingüístico y otros elementos propios de la lengua pueden transferirse entre las lenguas que la persona aprende. Estos conocimientos se complementan con las vías de acceso a los significados, es decir, a los conjuntos de sensaciones asociados a las palabras (Grinder \& Bandler, 1998; Krauss et al., 2000; Thornbury, 2002) para generar una red de apoyo de significados en la adquisición de la segunda lengua, utilizando todos los procedimientos asimilados durante el proceso de socialización (Butzkamm, 2000). Para Cummins (2006), la persona que aprende configura el conocimiento de la segunda lengua a través de puentes que construye entre las lenguas que conoce, las que está en proceso de conocer y las que experimenta y escucha en su diario vivir con otros, en un intercambio vivo y constante de saberes. Esto implica que los docentes deben facilitar que se generen las redes de apoyo de significados durante el proceso de aprendizaje y que ocurran intercambios sensoriales, cognitivos y lingüísticos para lograr que el estudiante desarrolle vocabulario en la segunda lengua y, por consiguiente, pierda el miedo a expresarse oralmente.

En torno a los antecedentes expuestos, se puede señalar que un proceso fundamental en el acceso a una lengua extranjera o una segunda lengua es la sensoriomotricidad, ya que a partir de ella se construyen los sistemas de comunicación (Allen, 2017; Goodwyn et al., 
2000). Al respecto, Herrera et al. (2014) y Allen (2017) señalan que las estrategias cognitivas derivadas de la modalidad visual-gestual permiten que personas sordas amalgamen información lingüística proveniente de distintas fuentes sensoriales (visual/kinésica/auditiva), para acceder al significado de las palabras en el segundo idioma. Este tipo de innovación metodológica plantea la necesidad de adoptar prácticas multimodales efectivas para lograr competencia comunicativa oral en contextos con escaso acceso a la segunda lengua.

En la actualidad, se postula que la activación de la producción oral en una lengua extranjera se enriquece al incorporar elementos como el movimiento y el tacto, en un proceso cognitivo activo y constructivo. Al respecto, Krauss et al. (2000) plantean que el conocimiento es multisensorial y multimedial y que las representaciones mentales requieren aportes de diversas modalidades sensoriales para un mejor acceso al léxico mental. Proponen el uso de la gestualidad para reducir el estrés causado por el esfuerzo de recordar una palabra en la segunda lengua. Para Anderson et al. (2001) y Omaggio (2001), las habilidades necesarias para la adquisición de una lengua extranjera se pueden asimilar rápidamente a través del sistema sensoriomotor del aprendiz, debido a que los conocimientos y los procesos cognitivos se conectan entre sí para generar redes de significados en el aprendizaje.

La valoración de las formas de aprender de los demás encierra una riqueza semántica y cultural que es necesario visibilizar y relevar, porque percibir la realidad desde otras perspectivas permite que la educación y la sociedad cambien, además de ofrecer alternativas de acceso a una lengua extranjera a partir de representaciones mentales variadas y atractivas. En este contexto, Allen (2017) propone el uso de la lengua de señas americana (ASL) en el aula de inglés como lengua extranjera como un puente viso-gestual que ayuda a la verbalización y comprensión del vocabulario de la segunda lengua, debido a que algunas señas de uso cotidiano poseen una naturaleza icónica; esto permite que exista congruencia entre la seña y su significado.

A partir de los antecedentes formulados, se propone usar señas de la lengua de señas chilena (LSCh) y deletreo manual de letras y fonemas como complemento visual y sensoriomotor para generar procesos de aprendizaje basados en la activación multisensorial y representaciones mentales multimodales para el aprendizaje de vocabulario oral 
en inglés en estudiantes oyentes de ESTP. Para investigar lo anterior, se planteó la siguiente hipótesis: "la estrategia didáctica para la enseñanza de vocabulario que incluye el uso de Lengua de Señas chilena y alfabeto manual produce un efecto en el vocabulario productivo oral de inglés en los estudiantes oyentes de ESTP".

\section{Método}

El diseño de la investigación se definió como cuasi experimental, con diseño de grupo control no equivalente (Campbell \& Stanley, 2011), debido a que ambos grupos de participantes eran grupos naturales (secciones conformadas previamente, pertenecientes a una misma especialidad). Se aplicó pretest y postest a ambos grupos. El grupo experimental recibió la intervención con la estrategia didáctica propuesta y el grupo control recibió enseñanza tradicional de vocabulario del idioma inglés.

\section{Participantes}

Las participantes fueron 60 mujeres oyentes entre 16 y 44 años, con una edad promedio de 27 años. Todas pertenecían a dos secciones de primer año de la carrera de Técnico en Educación Parvularia de un Centro de Formación Técnica de la ciudad de Santiago de Chile. Al momento de realizar el estudio, no existían varones matriculados en la carrera. El 100\% de las estudiantes realizó su enseñanza secundaria en establecimientos públicos o particulares subvencionados y no habían cursado módulos, talleres o cursos de inglés de forma independiente.

\section{Implementación de la estrategia didáctica}

La estrategia diseñada incluyó la incorporación de señas de la LSCh, deletreo manual, imágenes y pronunciación simultánea en inglés, en la enseñanza de vocabulario aislado y consistió en la instrucción y práctica sistemática de 120 palabras en inglés de uso frecuente del nivel A1 del Marco Común Europeo de Referencia para las lenguas. Las palabras 
se clasificaron en 4 categorías: ropa y accesorios, objetos cotidianos, objetos del hogar y profesiones y oficios. Cada categoría contenía 30 palabras.

Posteriormente, se elaboró el material visual para la implementación didáctica de la estrategia. Para cada categoría, se diseñó una serie de 30 diapositivas con fondo blanco (una por palabra), con la palabra escrita en inglés en fuente Arial 70, en color negro, en la esquina superior derecha de la diapositiva. Al costado izquierdo de la diapositiva se situó una imagen o fotografía ilustrativa del concepto. Por último, se insertó una imagen animada (en formato GIF) con la representación de la seña en LSCh en el sector inferior derecho de la diapositiva (Figura 1).

Figura 1. Ejemplo de material de la estrategia didáctica elaborada.

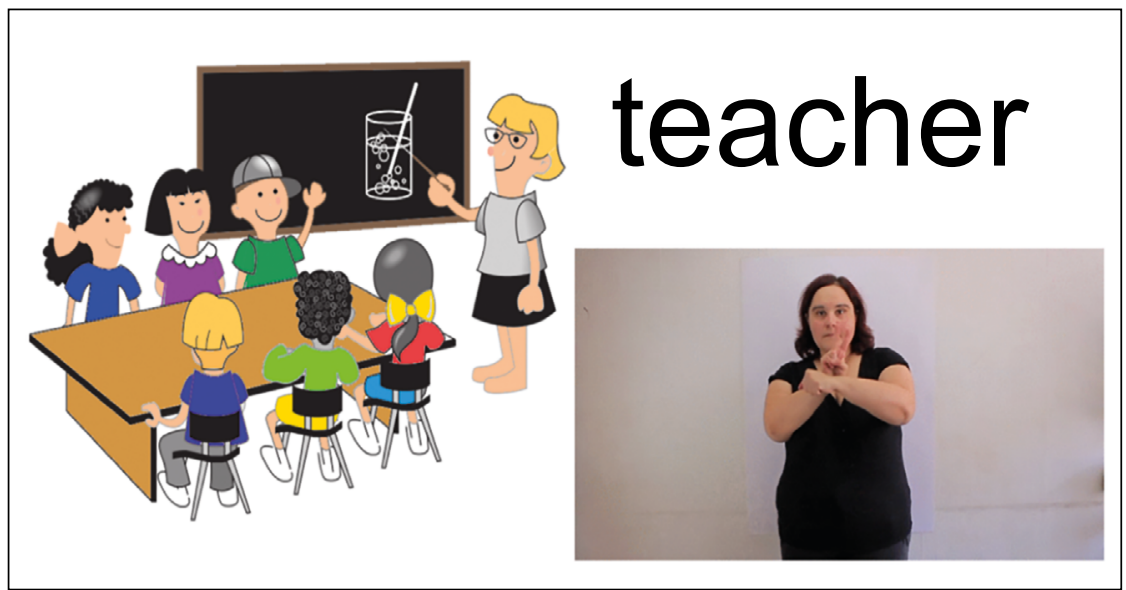

Fuente: Elaborado por las autoras.

La aplicación de la estrategia se realizó con el grupo experimental y, en ella, participó una profesora de inglés responsable de conducir las sesiones de trabajo. Durante la intervención, proyectó cuatro series de palabras, sus imágenes correspondientes en la pizarra e imágenes animadas de LSCh y guio el aprendizaje del vocabulario a través de deletreo manual (dactilología) y la pronunciación de la palabra junto con la ejecución de la seña en LSCh que aparecía en la diapositiva (ver Figura 2). La estrategia fue aplicada en cinco sesiones de 90 minutos cada una (10 horas pedagógicas). 
Figura 2. Esquema resumen estrategia didáctica.

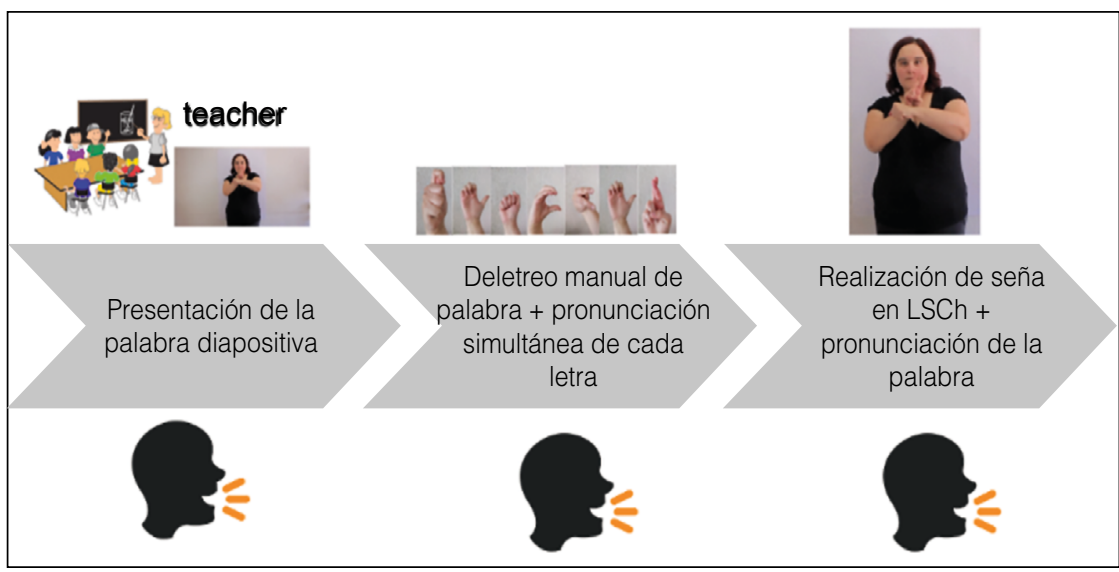

Fuente: Elaborado por las autoras.

Por su parte, el grupo control recibió instrucción con las mismas categorías de palabras, presentadas en diapositivas con imagen y palabra escrita, sin LSCh ni deletreo manual, limitando las sesiones a la repetición oral de las palabras que aparecían en las diapositivas.

\section{Instrumento}

El instrumento correspondió a una prueba oral de vocabulario productivo con imágenes diseñada especialmente para la investigación, cuya metodología de origen (Williams, 2007) es de uso extendido en evaluaciones orales de módulos de inglés en instituciones de ESTP. El instrumento se aplica de forma individual y evalúa la producción de vocabulario oral específico de nivel básico inicial (A1) por parte del estudiante. La aplicación de este instrumento tiene tres finalidades: 1) detectar el conocimiento previo de vocabulario de las participantes, 2) determinar, al finalizar la intervención, si hubo aumento de la producción de vocabulario oral del estudiante, y 3) contabilizar los tiempos de respuesta en cada ítem y apreciar si dichos tiempos disminuyen entre el pretest y el postest.

El instrumento posee dos formas (A y B) con cuatro láminas cada una. Cada lámina contiene 15 imágenes que representan palabras de vocabulario básico (60 palabras en total) pertenecientes a las categorías 
de ropa y accesorios, objetos de la vida cotidiana, vocabulario del hogar y profesiones y oficios. La tarea consiste en escoger una de las formas al azar, para posteriormente presentar cada una de las láminas al estudiante por separado.

El estudiante tiene 15 segundos para observar en silencio cada lámina y luego 45 segundos para pronunciar en voz alta —en inglésla mayor cantidad de palabras que aparecen en la lámina. Las respuestas se cotejan con pauta de corrección. Posteriormente, se contabilizan las respuestas correctas e incorrectas y se asigna puntaje (un punto por palabra correctamente pronunciada). También se contabilizan los tiempos de respuesta utilizados para cada grupo de palabras, dividiendo el tiempo total ocupado para responder cada ítem por el número total de palabras respondidas (correctas e incorrectas). Los tiempos de respuesta no afectan el puntaje final obtenido por el estudiante.

Para la validación del instrumento, se recurrió al juicio de expertos. Para los fines de esta investigación, se optó por cinco profesores, expertos en evaluación y/o currículum, que se desempeñaban en instituciones de enseñanza del inglés, colegios bilingües e instituciones de educación superior. Los jueces expertos revisaron y evaluaron el instrumento a partir de una rúbrica, que contempló las dimensiones de 1) construcción de los ítems del instrumento; 2) relación de los ítems con los contenidos y aprendizajes esperados; 3) coherencia entre el contenido a evaluar y lo evaluado; 4) contextualización de ítems; y 5) diseño gráfico. Los jueces consultados concluyeron de forma unánime que el instrumento medía lo propuesto.

Se realizó un análisis piloto con diez voluntarios adultos, cuyos resultados fueron sometidos a la prueba de confiabilidad del Coeficiente Alfa de Cronbach. El coeficiente alfa de Cronbach aplicado al instrumento entregó un valor de $\alpha=0,799$, que se aproxima a $\alpha=0,8$, lo que determina que el instrumento posee fiabilidad suficiente para ser aplicado.

El procesamiento de los datos se realizó usando el software estadístico SPSS versión 22.0 IBM. En primer lugar, se realizó un análisis descriptivo para obtener medidas de tendencia central, frecuencias y porcentajes para las variables cuantitativas, que posteriormente fueron sometidas a la prueba de normalidad Shapiro-Wilk. Para llevar a cabo 
la prueba de hipótesis, se aplicó la prueba estadística no paramétrica U de Mann-Whitney.

Para complementar los datos cuantitativos, se realizaron observaciones durante la realización de las pruebas para recoger impresiones, comentarios y actitudes de las participantes. Dichas observaciones se registraron en notas de campo que permitieron enriquecer el análisis de los datos cuantitativos.

\section{Resultados}

Las mediciones de pretest para ambos grupos (ver Tabla 1) indican que las participantes presentan medias de tiempos similares de recuperación de palabras de vocabulario en inglés, por lo que se puede aseverar que las participantes ocupan tiempos semejantes al recuperar palabras en inglés de su léxico mental para producirlas oralmente o toman el mismo tiempo en comprobar que las desconocen.

Al observar los resultados obtenidos en los tiempos de respuesta, se aprecia que ambos grupos disminuyen sus tiempos en el postest, pero la disminución del grupo experimental fue superior en las medias, mínimos y máximos. En el grupo experimental, la media de tiempo de respuesta disminuyó en 4,1 segundos, mientras que el grupo control disminuyó 2,59 segundos. Los mínimos y máximos del grupo control no presentaron mayor variación entre pretest y postest, mientras que, en el grupo experimental, los mínimos y máximos tuvieron una diferencia de 2 y 5,1 segundos entre pretest y postest, respectivamente. Las desviaciones estándar de los tiempos de respuesta se redujeron en ambos grupos entre pretest y postest, pero la reducción fue mayor en el grupo experimental. 
Tabla 1. Medidas de tendencia central, tiempos de respuesta.

\begin{tabular}{|c|c|c|c|}
\hline & & G. Con. & G. Exp. \\
\hline \multirow{4}{*}{ 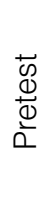 } & M & 12,75 & 12,05 \\
\hline & Min. & 4,40 & 5,60 \\
\hline & Max. & 37,50 & 21,90 \\
\hline & SD & 6,895 & 3,818 \\
\hline \multirow{4}{*}{$\begin{array}{l}\square \\
\Phi \\
\Phi \\
0 \\
0 \\
\square\end{array}$} & M & 10,19 & 7,95 \\
\hline & Mín. & 4,00 & 3,60 \\
\hline & Máx. & 37,50 & 16,80 \\
\hline & SD & 5,796 & 2,913 \\
\hline
\end{tabular}

$\mathrm{N}=30$ ambos grupos

Fuente: Elaborado por las autoras.

La Tabla 2 muestra que, en el postest del grupo control, la mayor concentración de frecuencias estuvo en los estudiantes cuyo tiempo de respuesta por palabra fue entre 0 y 10 segundos ( $f=19)$, que representa a un 63,3\% del grupo, mientras que un 36,6\% presenta un tiempo de respuesta mayor a 10 segundos. En cuanto al grupo experimental, el $86,7 \%$ de las estudiantes del grupo $(n=26)$ posee un tiempo de respuesta para la producción de una palabra entre 0 y 10 segundos, mientras que el $13,4 \%$ restante $(n=4)$ presenta un tiempo de respuesta mayor a 10 segundos en el postest. El efecto de la estrategia se observa en la reducción de los tiempos de respuesta del grupo experimental. El porcentaje de estudiantes que tardó 10 segundos o menos en rescatar palabras de su léxico mental para producirlas oralmente aumentó en un 56,7\% entre pretest y postest.

Tabla 2. Tabla de frecuencias y porcentajes de tiempos de respuesta, pre y postest

\begin{tabular}{|c|c|c|c|c|c|c|c|c|}
\hline & \multicolumn{4}{|c|}{ G. Control } & \multicolumn{4}{|c|}{ G. Experimental } \\
\hline & T. resp. & $F$ & $\%$ & $\%$ acum. & T. resp. & $f$ & $\%$ & $\%$ acum. \\
\hline \multirow{3}{*}{ 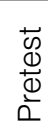 } & $<=10,00$ & 10 & 33,3 & 33,3 & $<=10,00$ & 9 & 30,0 & 30,0 \\
\hline & $10,01=<$ & 20 & 66,7 & 100,0 & $10,01=<$ & 21 & 70,0 & 100,0 \\
\hline & Total & 30 & 100,0 & & & 30 & 100,0 & \\
\hline
\end{tabular}




\begin{tabular}{|c|c|c|c|c|c|c|c|c|}
\hline & \multicolumn{4}{|c|}{ G. Control } & \multicolumn{4}{|c|}{ G. Experimental } \\
\hline \multirow{4}{*}{$\begin{array}{l}\mathbb{D} \\
\stackrel{\Phi}{00} \\
0 \\
0\end{array}$} & $<=10,00$ & 19 & 63,3 & 63,3 & $<=10,00$ & 26 & 86,7 & 86,7 \\
\hline & $10,01=<$ & 11 & 36,7 & 100,0 & $10,01=<$ & 4 & 13,4 & 100,0 \\
\hline & Total & 30 & 100,0 & 100,0 & & 30 & 100,0 & \\
\hline & $n=30$ & & & & $n=30$ & & & \\
\hline
\end{tabular}

Fuente: Elaborado por las autoras.

En la Tabla 3, se aprecia que el grupo experimental incrementó la media de respuestas correctas en 5,56 puntos entre pretest y postest, además de presentar una diferencia de 2,83 puntos respecto al grupo control en el postest. Dicha diferencia se refleja en la SD $=4,052$. Respecto a las respuestas incorrectas, el grupo experimental no presenta diferencias significativas. El grupo control presenta un aumento de 1,17 puntos entre pretest y postest. Las SD de ambos grupos se reducen entre pretest y postest. En cuanto a la omisión de palabras, se puede observar que ambos grupos omitieron menos palabras, pero la reducción de omisiones del grupo experimental fue mayor (5,83 puntos) respecto a la diferencia de pre y postest del grupo control, que fue de 1,2 puntos. La desviación estándar se reduce en el grupo control entre pre y postest, con una diferencia de 1,909, mientras que en el grupo experimental la desviación estándar aumenta 0,708 puntos entre pre y postest.

Tabla 3. Medidas de tendencia central de respuestas correctas, incorrectas y omitidas de instrumento de vocabulario oral

\begin{tabular}{|c|c|c|c|c|c|}
\hline & & \multicolumn{2}{|c|}{ Pretest } & \multicolumn{2}{|c|}{ Postest } \\
\hline & & M & SD & M & SD \\
\hline \multirow{3}{*}{ 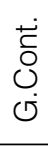 } & Correctas & 7,13 & 5,958 & 8,0 & 5,626 \\
\hline & Incorrectas & 3,63 & 3 & 4,8 & 2,797 \\
\hline & Omitidas & 48,4 & 9,343 & 47,2 & 7,434 \\
\hline \multirow{3}{*}{ 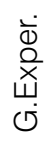 } & Correctas & 5,27 & 2,803 & 10,83 & 4,052 \\
\hline & Incorrectas & 2,57 & 2,096 & 2,80 & 1,919 \\
\hline & Omitidas & 52,2 & 4,131 & 46,37 & 4,839 \\
\hline
\end{tabular}

$\mathrm{N}=30$ ambos grupos

Fuente: Elaborado por las autoras. 
Realizados los análisis descriptivos, se efectuó la prueba de hipótesis (ver Tabla 4) en la que:

$\mathrm{H}_{0}:$ MeGEXP = MeGC (no existen diferencias en el postest entre las medianas de las variables de grupo experimental y control) y $\mathrm{H}_{\mathrm{i}}$ : MeGEX $\neq$ MeGC (existen diferencias entre las medianas de las variables de grupo experimental y control en el postest).

El nivel de significancia corresponde a $p=0,05$.

Tabla 4. Estadísticos de prueba U-Mann Whitney

\begin{tabular}{|l|c|c|c|c|}
\hline & \multicolumn{4}{|c|}{ Estadísticos de prueba (a) } \\
\hline & $\begin{array}{c}\text { R. Correctas } \\
\text { pretest }\end{array}$ & $\begin{array}{c}\text { R. Correctas } \\
\text { postest }\end{array}$ & $\begin{array}{c}\text { Tpo. } \\
\text { Respuesta } \\
\text { pretest }\end{array}$ & $\begin{array}{c}\text { Tpo. } \\
\text { Respuesta } \\
\text { postest }\end{array}$ \\
\hline U de Mann-Whitney & 394,000 & 244,000 & 415,500 & 281,500 \\
\hline Z & $-0,833$ & $-3,055$ & $-0,510$ & $-2,492$ \\
\hline $\begin{array}{l}\text { Sig. Asintótica } \\
\text { (bilateral) }\end{array}$ & 0,405 & 0,002 & 0,610 & 0,013 \\
\hline (a)=Variable de agrupación: GRUPO & & \\
\hline
\end{tabular}

Fuente: Elaborado por las autoras.

En la Tabla 4 se observa que en el número de palabras correctamente pronunciadas en el postest de los grupos experimental y control, el valor estadístico U corresponde a 415,5 con $\mathrm{p}=0,002<\mathrm{a}$ 0,05, lo que reporta que, en el postest, existen diferencias significativas entre las medianas de respuestas correctas. Respecto a los tiempos de respuesta del postest de los grupos experimental y control, el valor estadístico U corresponde a 244, con p=0,013 < a 0,05, lo que reporta que en el postest existen diferencias significativas entre las medianas de los grupos.

El análisis inferencial reporta que, al comparar los niveles de vocabulario productivo oral en inglés antes y después de la implementación de la estrategia didáctica en estudiantes de ESTP, se apreció un efecto positivo en la producción oral de vocabulario en el grupo experimental, cuyo aumento del número de palabras pronunciadas correctamente y reducción de tiempos de respuesta en relación con el pretest fueron mayores que los del grupo control. 
Las notas de campo recogidas en los diferentes momentos del estudio permiten complementar algunos aspectos del análisis cuantitativo, como los factores emocionales que influyen en la oralización del vocabulario en la lengua extranjera. Al comenzar el pretest, en ambos grupos se observa que la mayoría de las participantes emitían comentarios como "no sé", "no me acuerdo", "no tengo idea", "no sé nada", "no tuve inglés en el colegio", "parece que esto es, pero no estoy segura..." y repiten esos comentarios al finalizar el tiempo destinado para la prueba. Se registra que algunas estudiantes golpetean la mesa con los dedos, se rascan la cabeza o mueven sus piernas cuando les toca el turno de pronunciar.

En cuanto a los errores de pronunciación, se observa que las participantes castellanizan las palabras para realizar una pseudo pronunciación en inglés ("cantant" /knntwnt/ en vez de "singer" /sinər/). Algunas participantes omiten ítems completos y demoran entre 6 y 10 segundos en declarar que desconocen el vocabulario presentado.

Durante la intervención, las participantes del grupo experimental muestran entusiasmo por aprender los contenidos propuestos de la forma planteada, algunas manifiestan satisfacción al aprender algunas señas de LSCh y contar con herramientas básicas para comunicarse con personas sordas y a la vez aprender vocabulario en inglés.

En cuanto a aspectos motores, las estudiantes presentan dificultades para realizar la configuración manual (deletreo manual) de algunas letras, especialmente D, F, K, Q y T, las que fueron superando al transcurrir las sesiones de trabajo. Para superar esta dificultad, se incorporaron ejercicios de relajación para manos y dedos al iniciar la ejercitación, repasando el alfabeto manual con la mano derecha y la izquierda, acompañada con la pronunciación en inglés de cada letra. La realización del deletreo manual implicó algunas dificultades de las estudiantes como: confundir la pronunciación de las vocales A /ei/, E /i/, I /ai/ y las consonantes G/dz/ y J /dzi/. Durante las últimas sesiones de la intervención realizada con el grupo experimental, las estudiantes logran pronunciar correcta y autónomamente los fonemas, acompañados del deletreo manual correspondiente.

Finalmente, en el postest, las estudiantes del grupo experimental no emitieron comentarios de ningún tipo al presentar las palabras, observaban las láminas y responden, la mayoría indica con el dedo 
la imagen y pronuncian correctamente. Algunas articulan la seña en LSCh al observar la imagen y pronuncian la palabra en voz alta en el tiempo estimado para ello. Se observan más relajadas que en el pretest. Igualmente, las participantes del grupo control se observan más confiadas que en el pretest e intentan pronunciar más palabras, pero continúan fallando al pronunciarlas.

\section{Discusión}

Los resultados obtenidos concuerdan con lo propuesto por Krauss et al. (2000) respecto a que el canal sensoriomotor facilita la recuperación de vocabulario al momento de oralizar. El porcentaje de participantes del grupo experimental que pronunciaron correctamente cinco palabras o menos en el pretest disminuyó, aumentando a un $90 \%$ las estudiantes que pronuncian correctamente más de seis palabras en el postest, mientras que en el postest realizado al grupo control, solo un $66 \%$ de las estudiantes pronunció correctamente seis palabras o más. Los resultados del estudio concuerdan con lo propuesto por Hotstetter et al. (2007), que el uso de estrategias multimodales (y en específico, el uso de los movimientos manuales) en la producción de vocabulario oral facilita la capacidad de oralización. Este tipo de estrategia se alinea con planteamientos que ya son ampliamente utilizados en la enseñanza del inglés, como el de Total Physical Response, que, al incorporar movimientos del cuerpo al discurso hablado, ayuda a disminuir el estrés del acto de aprender y modificar los filtros afectivos negativos que bloquean la disposición del estudiante para oralizar en inglés (Allen, 2017; Krashen, 2002; Hotstetter det al., 2007; Richards \& Rodgers, 2001).

Igualmente, es necesario reflexionar respecto del contexto educativo de las participantes, en cuanto que todas realizaron estudios secundarios en colegios de dependencia municipal (pública) y particular subvencionada. Este dato puede ayudar a comprender que el acceso al aprendizaje de la lengua inglesa del segmento socioeconómico que ingresa a la ESTP presenta mayores desafíos y dificultades (British Council, 2015; Kormos \& Kiddle, 2013; Ministerio de Educación de Chile, 2013a, 2013b). Estas dificultades podrían atribuirse en parte a las 
metodologías empleadas mayoritariamente en la enseñanza del inglés en la educación secundaria, que desalientan al aprendizaje de vocabulario para promover la producción oral; incluso más, desmotivan al estudiante y lo vuelven temeroso de hablar en inglés (British Council, 2015; Krashen, 2002; McBride, 2009; Riasati \& Rahimi, 2018; Vera, 2008).

En la aplicación de la estrategia, la repetición continua del deletreo manual y los fonemas, la seña en LSCh, la pronunciación de la palabra y el uso de imágenes (Allen, 2017; Herrera et al., 2014; Syukri \& Humaera, 2019) ayudaron a reducir la tensión y timidez de las estudiantes en las sesiones de trabajo. Al respecto, resulta interesante comprobar que varias estudiantes del grupo experimental, durante el postest, realizaron señas antes de pronunciar las palabras en inglés. Esto refleja el uso del canal kinestésico como forma de acceder al léxico mental (Krauss et al., 2000; Hotstetter et al., 2007) y las interrelaciones entre elementos abstractos de las lenguas que la persona aprende, formulada por Cummins (2002).

\section{Conclusiones}

La enseñanza del inglés en Chile se encuentra actualmente en un proceso de promoción curricular (Ministerio de Educación de Chile, 2019), al igual que la lengua de señas chilena, que experimenta un proceso de valorización social. El cambio de paradigma respecto de las lenguas minoritarias ha permitido conocer y difundir el valor lingüístico y cultural de la diversidad humana (Bauman \& Murray, 2014). Estos procesos de cambio social permiten avanzar en la generación de estrategias de enseñanza a partir del reconocimiento de la riqueza multisensorial en el diseño instruccional y su aporte a la generación de experiencias de aprendizaje significativas.

Una de las razones que lleva a proponer esta estrategia multisensorial para la enseñanza de vocabulario en inglés, utilizando herramientas lingüísticas de la comunidad de sordos, fue la posibilidad de diversificar las vías de acceso al vocabulario oral, considerando las características de las estudiantes y las complejidades que presenta la enseñanza del idioma inglés en Chile. En este sentido, la estrategia 
permitió a las estudiantes apropiarse del vocabulario usando todos los sentidos (Castro \& Rodríguez, 2017; Grinder \& Bandler, 1998; Syukri \& Humaera, 2019). La valoración del potencial viso-gestual de la LSCh como herramienta de integración multisensorial y la consiguiente diversificación de metodologías para la enseñanza de vocabulario oral en inglés como lengua extranjera en estudiantes adultas permitió mejorar su aprendizaje (Allen, 2017; Undurraga, 2007) y estimular la producción oral de vocabulario en la lengua extranjera. Los resultados del estudio permiten afirmar que la estrategia afecta de forma significativa la producción oral de vocabulario en inglés.

Por otra parte, los resultados de las pruebas de vocabulario realizadas también confirman que la producción oral de vocabulario en inglés de las estudiantes es deficiente, resultados que van en congruencia con las mediciones estandarizadas realizadas en adultos (English First, 2019; British Council, 2015) y con las mediciones SIMCE de inglés (Ministerio de Educación de Chile, 2013b).

Es fundamental, para futuras investigaciones, tomar en consideración que la voluntad de oralizar (sea en la lengua propia o en una segunda lengua) es un fenómeno multifactorial, que incluye aspectos de personalidad del sujeto que aprende, su contexto socioeconómico, motivación, entre otros (Kormos \& Kiddle, 2013; MacIntyre et al., citados en Riasati \& Rahimi, 2018).

Es tarea del profesor tener en cuenta esos factores y proveer múltiples medios en el diseño de sus estrategias para favorecer la producción oral (Castro \& Rodríguez, 2017; Syukri \& Humaera, 2019), flexibilizando y facilitando el uso de todos los sentidos para contribuir al acto de habla en una lengua extranjera. Si se quiere promover aprendizajes que permitan autonomía oral comunicativa al estudiante de ESTP, deben proveerse herramientas atractivas que le permitan vencer sus aprensiones y compensar sus deficiencias previas (Riasati \& Rahimi, 2018).

La educación chilena experimenta un cambio paradigmático que pretende alcanzar el ideal de la inclusión y respeto a la diversidad en las aulas (Ministerio de Educación de Chile, 2018). Para lograrlo, es imprescindible comprender que tanto la inclusión como el respeto a la diversidad deben traducirse en acciones y modelos educativos inclusivos que consideren el intercambio cultural e identitario (UNESCO, 2008) y enriquecer las formas de enseñar y aprender a través de la valoración 
de la diversidad lingüística, cultural y funcional. Para lograr dichos objetivos, los diseños instruccionales deben ser coadyuvantes en la concreción de la inclusión educativa en todas las áreas del conocimiento.

Para una educación efectivamente inclusiva es imprescindible transformar las prácticas educativas y diseñar nuevas estrategias de aprendizaje pertinentes, significativas, contextualizadas y participativas (Ministerio de Educación de Chile, 2009), utilizando todas las redes de apoyo cognitivo que se puedan entregar (Castro \& Rodríguez, 2017; Grinder \& Bandler, 1998; Undurraga, 2007). Por último, resulta fundamental que los docentes exploren y experimenten variadas didácticas para la enseñanza del inglés, teniendo en cuenta que no existen metodologías completamente efectivas y aplicables a cualquier contexto (McBride, 2009), sino que las más efectivas son las que emergen de las interacciones, de la experiencia en el aula y del conocimiento mutuo generado entre ellos (Richards \& Rodgers, 2001). Por ello, es importante enseñar y aprender con todos los sentidos de la experiencia del otro, de sus metodologías diversas, vivencias y hallazgos (Lohaus-Reyes, 2019), para legitimar, visibilizar y valorizar lo que ya está cerca, de las formas de enseñar y de vivir el mundo.

En cuanto a las proyecciones de la investigación, se sugiere seguir profundizando el estudio respecto al efecto a largo plazo de esta estrategia, dada la complejidad del contexto donde se llevó a cabo. Considerando lo anterior, se propone realizar modelos cuasi experimentales con mayor control de amenazas del que fue posible en esta investigación y poder realizar un seguimiento a la evolución de la producción oral en inglés de los estudiantes intervenidos, ampliando el vocabulario que se trabajó.

Entre las limitaciones del estudio, se asume que este se desarrolló con grupos naturales en un contexto específico, limitando la generalización de los resultados obtenidos. Asimismo, se asume que la intervención con la estrategia fue acotada tanto en tiempos como en contenidos y no se pretendió hacer un seguimiento a través del tiempo de las participantes y los grupos. 


\section{Referencias}

Allen, V. (2017). American Sign Language as a bridge to English: A handbook for hearing teachers of hearing students. Paperback.

Anderson, L. W., Krathwohl, D. R., Airasian, P., Cruikshank, K., Mayer, R., Pintrich, P., \& Wittrock, M. (2001). A taxonomy for learning, teaching and assessing: A revision of Bloom's taxonomy. Longman Publishing.

Bauman, H-D., \& Murray, J. (Eds.) (2014). Deaf gain: Raising the stakes for human diversity. Minnesota Press.

Brown, D. (2000). Principles of language learning and teaching. Pearson Education.

British Council. (2015). English in Chile. An examination of policy, perceptions and influencing factors. https://ei.britishcouncil.org/sites/default/files/ latin-america-research/English\%20in\%20Chile.pdf

Butzkamm, W. (2003). We only learn language once. The role of the mother tongue in FL classrooms: Death of a dogma. The Language Learning Journal, 28(1), 29-39. https://doi.org/10.1080/09571730385200181

Campbell, D., \& Stanley, J. (2011). Diseños experimentales y cuasiexperimentales en ciencias sociales. Amorrortu.

Castro, R., \& Rodríguez, F. (2017). Diseño universal para el aprendizaje y co-enseñanza. Estrategias pedagógicas para una educación inclusiva. RIL Editores.

Cummins, J. (2002). Lenguaje, poder y pedagogía: niños y niñas bilingües entre dos fuegos. Ediciones Morata.

Cummins, J. (2006). The relationship between ASL proficiency and English academic development: A review of the research. Ponencia presentada en Workshop Challenges, Opportunities, and Choices in Educating Minority Group Students. Hamar University College, Noruega.

Echeita, G., \& Ainscow, M. (2011). La educación inclusiva como derecho. Marco de referencia y pautas de acción para el desarrollo de una revolución pendiente. Tejuelo, 12, 26-46.

English First (2019). English proficiency index report. https://www.ef.com/_/ / media/centralefcom/epi/downloads/full-reports/v9/ef-epi-2019-english.pdf

García, O., \& Cole, D. (2014). Deaf gains in the study of bilingualism and bilingual education. En H-D. Bauman \& J. Murray (Eds.), Deaf gain: Raising the stakes for human diversity, (95-111). University of Minnesota Press. 
Goodwyn, S. W., Acredolo, L. P., \& Brown, C. A. (2000). Impact of symbolic gesturing on early language development. Journal of Nonverbal behavior, 24(2), 81-103. https://doi.org/10.1023/A:1006653828895

Grinder, J., \& Bandler, R. (1998). De sapos a príncipes. Cuatro Vientos.

Herrera, V., Puente, A., \& Alvarado, J. M. (2014). Visual learning strategies to promote literacy skills in prelingually deaf readers. Revista Mexicana de Psicología, 31(1), 1-10.

Hostetter, A. B., Alibali, M. W., \& Kita, S. (2007). Does sitting on your hands make you bite your tongue? The effects of gesture prohibition on speech during motor descriptions. En Proceedings of the Annual Meeting of the Cognitive Science Society. https://escholarship.org/content/ qt6g02f7bv/qt6g02f7bv.pdf

Kormos, J., \& Kiddle, T. (2013). The role of socio-economic factors in motivation to learn English as a foreign language: The case of Chile. System, 41(2), 399-412. https://doi.org/10.1016/j.system.2013.03.006

Krashen, S. (2002). Theory versus practice in language training. En V. Zamel \& R. Spack. (Eds.), Enriching ESOL pedagogy: Readings and activities for engagement, reflection, and inquiry (pp. 211-228). Routledge.

Krauss, R., Chen, Y., \& Gottesman, R. (2000) Lexical gestures and lexical access: a process model. En D. McNeill. (Ed.), Language and Gesture, (pp. 261-283). Cambridge University Press. https://doi.org/10.1017/ CB09780511620850.017

Kumaravadivelu, B. (2003). Beyond methods: Macrostrategies for language teaching. Yale University Press.

Larsen-Freeman, D. (2004). Teaching language: From grammar to grammaring. Thomson Heinle.

Lohaus-Reyes, M. F. (2019). Curricular decolonization from epistemologies of diversity. Transnational Curriculum Inquiry, 16(1), 74-80.

McBride, K. (2009). Percepciones estudiantiles sobre las técnicas utilizadas en la enseñanza del inglés como lengua extranjera. Universum (Talca), 24(2), 94-112. https://doi.org/10.4067/S0718-23762009000200006

McKay, S. (2003). Teaching English as an international language: The Chilean context. ELT journal, 57(2), 139-148. https://doi.org/10.1093/ elt/57.2.139

Méndez, M. G., \& Peña, A. (2013). Emotions as learning enhancers of foreign language learning motivation. Profile Issues in Teachers' Professional Development, 15(1), 109-124. http://www.scielo.org.co/scielo. 
php?script=sci_arttext\&pid=S1657-07902013000100008\&lng=en\&tlng=en

Ministerio de Educación de Chile. (2009). Criterios y orientaciones de flexibilización del curriculum. Para dar respuesta a la diversidad en los distintos niveles y modalidades de enseñanza. https://especial.mineduc.cl/ wp-content/uploads/sites/31/2016/08/Criterios_Orientaciones_Flexibilizacion_Curricular-2009.pdf

Ministerio de Educación de Chile. (2013a). Resultados SIMCE inglés III medio 2012 [Archivo PDF]. http://www.educacion2020.cl/sites/default/ files/resultados_iii_medio_simce_ingles_2012.pdf

Ministerio de Educación de Chile. (2013b). Informe de resultados SIMCE inglés 2012 para docentes y directivos. Unidad de Currículum y Evaluación. [Archivo PDF]. http://archivos.agenciaeducacion.cl/biblioteca_digital_historica/resultados/2012/ire3m_2012.pdf

Ministerio de Educación de Chile. (2016). Bases curriculares formación diferenciada técnico profesional. [Archivo PDF]. https://www.curriculumnacional.cl/614/articles-70892_bases.pdf

Ministerio de Educación de Chile. (2019). Bases curriculares $3^{\circ}$ y $4^{\circ}$ medio. [Archivo PDF]. https://curriculumnacional.mineduc.cl/614/articles-91414_bases.pdf

Omaggio, A. (2001). Teaching language in context. Heinle and Heinle.

Riasati, M. J., \& Rahimi, F. (2018). Situational and individual factors engendering willingness to speak English in foreign language classrooms. Cogent Education, 5(1). 1513313https://doi.org/10.1080/23311 86X.2018.1513313

Richards, J., \& Rodgers, T. (2001). Approaches and methods in language teaching. Cambridge University Press. https://doi.org/10.1017/CB0978051 1667305

Syukri, S., \& Humaera, I. (2019). Gaining motivation on English learning for special need students using flashcards, foldable books and posters in EFL context. Langkawi: Journal of The Association for Arabic and English, 5(2), 91-101. https://doi.org/10.31332/lkw.v5i2.1303

Thornbury, S. (2002). How to teach vocabulary. Pearson Education limited.

Undurraga, C. (2007). ¿Cómo aprenden los adultos? Una mirada psicoeducativa. Ediciones UC.

UNESCO. (2008). La educación inclusiva: el camino hacia el futuro. 48 Reunión de la Conferencia Internacional de Educación. [Archivo PDF] http:// 
www.ibe.unesco.org/fileadmin/user_upload/Policy_Dialogue/48th_ ICE/General_Presentation-48CIE-4_Spanish_.pdf

Vera,F.(2008). Estado delartedela profesión deprofesor deinglés: ¿Quéocurre en Chile? Nodo educativo. Universidad Tecnológica Metropolitana. http:// www.utemvirtual.cl/nodoeducativo/wp-content/uploads/2009/

122 03/art_fvera.pdf

Vertebral Chile. (2018). Información estadística. http://vertebralchile.cl/informacion-estadistica/

Williams, K. T. (2007). Expressive vocabulary test (2nd Ed.). Pearson Assessments. https://doi.org/10.1037/t15094-000

Zabalza, M. A. (2003). Las competencias docentes del profesorado universitario. Calidad y desarrollo profesional. Narcea.

Zajda, J. (2018). Globalisation and education reforms: Paradigms and ideologies. En J. Zajda. (Ed.) Globalisation and education reforms, 1-14. Springer. https://doi.org/10.1007/978-94-024-1204-8

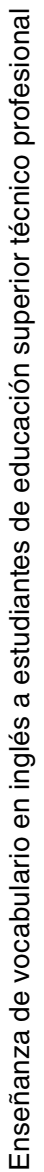

年 